University of South Carolina

Scholar Commons

3-9-2009

\title{
Macrospin model to explain the absence of preswitching oscillations in magnetic tunnel junctions: Fieldlike spin-transfer torque
}

\author{
Samir Garzon \\ University of South Carolina - Columbia \\ Yaroslaw Bazaliy \\ University of South Carolina - Columbia \\ Richard A. Webb \\ University of South Carolina - Columbia, webbra@mailbox.sc.edu \\ Mark Covington \\ Shehzaad Kaka
}

See next page for additional authors

Follow this and additional works at: https://scholarcommons.sc.edu/phys_facpub

Part of the Physics Commons

Publication Info

Published in Physical Review B, Volume 79, Issue 10, 2009, pages 100402-1-100402-4.

Garzon, S., Bazaliy, Y., Webb, R.A., Covington, M., Kaka, S., and Crawford, T.M. (2009). Macrospin model to explain the absence of preswitching oscillations in magnetic tunnel junctions: Fieldlike spin-transfer torque. Physical Review B, 79(10), 100402-1 - 100402-4. doi: 10.1103/PhysRevB.79.100402 (C) 2009 The American Physical Society.

This Article is brought to you by the Physics and Astronomy, Department of at Scholar Commons. It has been accepted for inclusion in Faculty Publications by an authorized administrator of Scholar Commons. For more information, please contact digres@mailbox.sc.edu. 


\section{Author(s)}

Samir Garzon, Yaroslaw Bazaliy, Richard A. Webb, Mark Covington, Shehzaad Kaka, and Thomas M. Crawford 


\title{
Macrospin model to explain the absence of preswitching oscillations in magnetic tunnel junctions: Fieldlike spin-transfer torque
}

\author{
Samir Garzon, ${ }^{1}$ Yaroslaw Bazaliy, ${ }^{1,2}$ Richard A. Webb, ${ }^{1}$ Mark Covington, ${ }^{3}$ Shehzaad Kaka, ${ }^{3}$ and Thomas M. Crawford ${ }^{1}$ \\ ${ }^{1}$ Department of Physics and Astronomy and USC Nanocenter, University of South Carolina, Columbia, \\ South Carolina 29208, USA \\ ${ }^{2}$ Institute of Magnetism, National Academy of Sciences, Kyiv 03142, Ukraine \\ ${ }^{3}$ Seagate Research, 1251 Waterfront Place, Pittsburgh, Pennsylvania 15222, USA
}

(Received 19 October 2008; published 9 March 2009)

\begin{abstract}
We show that the absence of preswitching oscillations ("incubation delay") in magnetic tunnel junctions can be explained within the macrospin model by a sizable fieldlike component of the spin-transfer torque. It is further suggested that measurements of the voltage dependence of tunnel junction switching time in the presence of external easy axis magnetic fields can be used to determine the magnitude and voltage dependence of the fieldlike torque.
\end{abstract}

DOI: 10.1103/PhysRevB.79.100402

PACS number(s): 72.25.Ba, 85.75.-d

A spin polarized electric current can transfer spin angular momentum to a magnetic material, generating a torque that can induce magnetization dynamics and even magnetization reversal. ${ }^{1,2}$ While extensive measurements have tested the validity and limitations of the macrospin model with Slonczewski's spin-transfer torque in metallic spin valves, recent experiments with magnetic tunnel junctions (MTJs) (Refs. 3-6) have observed an additional "fieldlike" or "perpendicular" spin torque. The existence of a fieldlike torque was predicted for metallic spin valves ${ }^{7-9}$ but shown to be smaller than Slonczewski's "parallel" torque. ${ }^{10,11}$ For magnetic tunnel junctions, however, it was predicted that both torques could have similar magnitudes and that the fieldlike torque would have a quadratic dependence on voltage. ${ }^{12,13}$ The observed fieldlike torques generally agree with theoretical predictions, but some controversies remain. For example, measurements in the frequency domain at low voltages ${ }^{4,5}$ and measurements of switching currents at large voltages ${ }^{6}$ report contradictory signs of the fieldlike term. This suggests that further theoretical analysis and experimental investigation are necessary to fully understand the origin and the functional form of this torque.

Here we report that the fieldlike torque can explain the absence of the preswitching oscillations ("incubation delay") found by Devolder et al. ${ }^{14}$ in MTJs. This observation could not be described within a macrospin model of magnetization reversal based on Slonczewski's spin-transfer torque alone. Such a model predicts that pumping of the ferromagnetic resonance mode produces increasing oscillations in the resistance before switching. ${ }^{15,16}$ However, by including the effects of a fieldlike spin torque term within a macrospin model we are able to reproduce the main features of the observed magnetization reversal: (i) a slow regular change in the resistance without oscillations preceding the switching, (ii) decaying oscillations of the resistance after switching, and (iii) similarity between magnetization reversal curves shifted so as to align their switching times (in our case the switching times $t_{S}$ are distributed between 0 and $10 \mathrm{~ns}$ ). In addition, we propose time-domain experiments which could be used to measure the magnitude of the fieldlike spin torque term and its voltage dependence, settling the mentioned sign issues and motivating further theoretical investigation.

We consider a macrospin model corresponding to a rectangular MTJ [Fig. 1(a)] with nominal free layer dimensions $100 \times 300 \times 2.5 \mathrm{~nm}^{3}$, saturation magnetization $4 \pi M_{S}$ $=4.4 \mathrm{kG}$, easy-axis anisotropy field $2 K / M_{S}=H_{K}=80 \mathrm{Oe}$, dipole field coupling to the reference layer $H_{D}=28 \mathrm{Oe}$ favoring the $\mathrm{P}$ state, and with parallel (P) and antiparallel (AP) resistances $R_{P}=286 \Omega$ and $R_{\mathrm{AP}}=364 \Omega$, respectively, as quoted in Ref. 14. We use an easy-plane anisotropy field $2 K_{P} / M_{S}=H_{P}=4280$ Oe which arises due to the geometrical anisotropy of the free layer. Within the macrospin model, the energy density of the nanomagnet, ${ }^{15}$
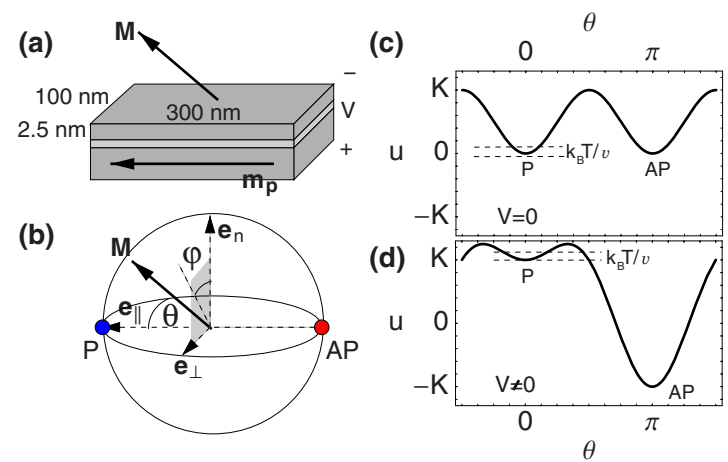

FIG. 1. (Color online) (a) Schematic of rectangular MTJ showing the free layer magnetization $\mathbf{M}$ at an arbitrary orientation, and the reference layer magnetization $\mathbf{m}_{\mathbf{P}}$ pinned along the easy axis. The polarity of the voltage $V$ applied across the sample is shown. (b) Unit sphere describing the possible orientations of M. Unit vectors normal to the sample $\left(\mathbf{e}_{\mathbf{n}}\right)$ and along the easy $\left(\mathbf{e}_{\|}\right)$and hard axes $\left(\mathbf{e}_{\perp}\right)$ are shown. The orientation of $\mathbf{M}$ is described by $\theta$, the angle between the free and pinned magnetizations, and $\varphi$, the angle between $\mathbf{e}_{\mathbf{n}}$ and the projection of $\mathbf{M}$ onto the $\mathbf{e}_{\mathbf{n}}-\mathbf{e}_{\perp}$ plane. Points $\mathrm{P}$ and $\mathrm{AP}$ are the equilibrium positions of $\mathbf{M}$ along the easy axis. (c), (d) Schematics of MTJ energy density landscape (c) before and (d) during a voltage pulse, for $\mathbf{M}$ in plane $(\varphi=\pi / 2)$, where $K$ is the easy-axis anisotropy energy density, $k_{B} T$ is the thermal energy at temperature $T$, and $v$ is the free layer volume. In (d) the fieldlike spin torque lowers the energy barrier allowing magnetization switching via a combination of Slonczewski's spin torque and random fluctuations. 


$$
u=-K\left(\mathbf{M} \cdot \mathbf{e}_{\|}\right)^{2}-M_{S}\left(H_{D}+H_{\|}\right) \mathbf{M} \cdot \mathbf{e}_{\|}+K_{P}\left(\mathbf{M} \cdot \mathbf{e}_{n}\right)^{2},
$$

is due to uniaxial anisotropy, magnetic fields, and uniplanar anisotropy, respectively (see the caption to Fig. 1 for definitions of field and unit vector components). The magnetization orientation of the free layer, given by the unit vector $\mathbf{M}$, is described by the angles $\theta$ and $\varphi$ defined in Fig. 1(b). In the absence of external fields and voltage and ignoring any magnetic coupling between the free and pinned layers, the equilibrium positions of $\mathbf{M}$ lie on the easy axis. A schematic of the energy density profile at $\varphi=\pi / 2$, i.e., for in-plane $\mathbf{M}$, is shown in Fig. 1(c).

We generate a Maxwell-Boltzmann distribution of 1000 initial orientations of $\mathbf{M}$ corresponding to a temperature of $300 \mathrm{~K}$. The time evolution of the ensemble of trajectories is found by solving the stochastic Landau-Lifshitz-Gilbert (LLG) equation in the presence of both Slonczewski's ${ }^{1}$ and fieldlike ${ }^{6}$ spin torque terms,

$$
\tau=a_{J} \mathbf{M} \times\left(\mathbf{M} \times \mathbf{m}_{\mathbf{P}}\right)+b_{J} \mathbf{M} \times \mathbf{m}_{\mathbf{P}},
$$

using the energy density described above. The effects of thermal fluctuations during the evolution of $\mathbf{M}$ are incorporated into the LLG equation by using Langevin fields. ${ }^{17,18}$ Each repetition of our simulation randomly chooses an initial orientation of $\mathbf{M}$ and evolves it stochastically, closely imitating a real experiment where each measurement tracks a trajectory with a distinct initial orientation and subject to different thermal fluctuations. We describe the angle dependence of the MTJ resistance by $R(\theta)=R_{P}+\left(R_{\mathrm{AP}}-R_{P}\right) \sin ^{2} \theta / 2$. Although the results shown here are for an angle-independent efficiency, $g(\theta, p)=\eta$, similar results were obtained by including Slonczewski's MTJ efficiency, $g(\theta, p)=p$ / $\left(2+2 p^{2} \cos (\theta)\right),{ }^{19}$ where $p$ is the spin polarization of the tunneling electrons. As proposed by $\mathrm{Li}$ et $a l .{ }^{6}{ }^{6}$ we use $b_{J}$ $=\epsilon|V| a_{J}$, where $V$ is the voltage and $\epsilon$ controls the relative amplitude of the two spin torque terms. Reference 6 estimates $\epsilon \sim 1 V^{-1}$ for typical magnetic materials. We model the voltage waveform as a step with 55 ps risetime. By appropriately choosing the values of $\epsilon, \eta$, and the damping, $\alpha$, it is possible to obtain a situation where (i) the fieldlike spin torque is small enough so that the $\mathrm{P}$ state is still stable and a large majority of the thermally distributed initial orientations of $\mathbf{M}$ are within the stability region of $\mathbf{P}$, as shown in Fig. 1(d), and (ii) the Slonczewski spin torque is too weak to induce magnetization reversal by itself. Under these conditions, it is the combination of Slonczewski's spin torque together with thermal fluctuations which eventually push the magnetization over the barrier, which has been lowered by the fieldlike spin torque. From the values of $R_{P}$ and $R_{\mathrm{AP}}$ we obtain a zero bias tunneling magnetoresistance (TMR) $\approx 27 \%$, from which the spin polarization $p \approx 34 \%$ and efficiency $\eta \approx 0.15$ can be obtained. ${ }^{20}$

We first find the time evolution of $\mathbf{M}$ for $\eta=0.15, \alpha$ $=0.02, \epsilon=1.7 \mathrm{~V}^{-1}$, and $V=1 \mathrm{~V}$. We generate 1000 trajectories such as the one shown in Fig. 2(a). Then we randomly pick ten of them, one by one, with the restriction that any picked trajectory has to have its switching time clearly spaced from all previously picked trajectories. This process ensures that the set is representative of the whole ensemble.
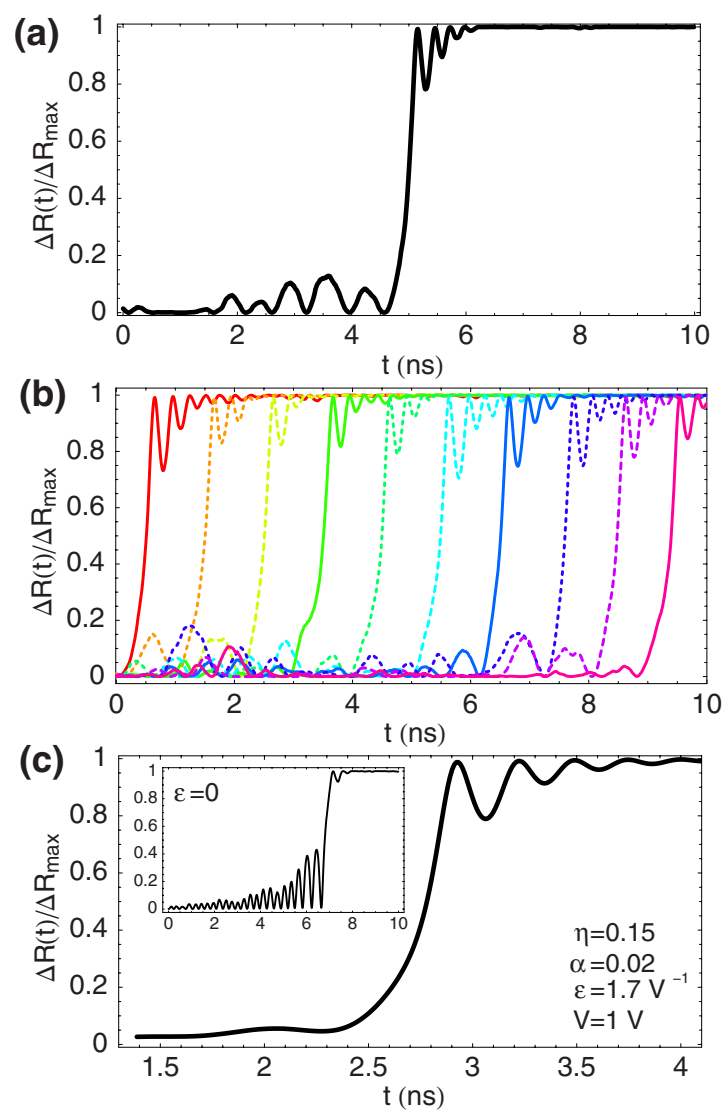

FIG. 2. (Color online) (a) Normalized change in resistance as a function of time for a randomly chosen trajectory. (b) Same for a set of trajectories with switching times $t_{S}$ distributed between 0 and 10 ns. (c) Average of all resistance traces with $\left|t_{S}-\overline{t_{S}}\right| \leq \delta t_{S}$ after aligning their switching time with the average value $\overline{t_{S}}=2.75 \mathrm{~ns}$. Inset: switching via Slonczewski's spin torque only $(\epsilon=0)$. All other parameters are kept constant.

The time dependence of the resistance for the selected trajectories is shown in Fig. 2(b) (compare with Fig. 4 in Ref. 14). We observe (i) a slow initial increase in the resistance, (ii) random preswitching fluctuations and reproducible postswitching ringing, and (iii) similarity in the behavior of M trajectories shifted so as to align their switching times. Furthermore, even though in some traces the transition from a slow resistance increase to a fast switch is subtle, clear transitions at about $0.2 \Delta R_{\max }$ are observable in many of the traces. We note that this value corresponds to the resistance at the top of the energy barrier [Fig. 1(d)] separating the P and AP states. The average switching time for the ensemble of trajectories is $\overline{t_{S}}=2.8 \mathrm{~ns}$, close to the value measured in Ref. 14 at $1.1 \mathrm{~V}\left(\overline{t_{S}}=2.5 \mathrm{~ns}\right)$ but smaller than what they observed at $1 \mathrm{~V}\left(\overline{t_{S}}=5.3 \mathrm{~ns}\right)$. However, complete numerical agreement with the experimental results is not expected since the average switching time depends on the function $g(\theta, p)$ and the value of $\epsilon$ used, which are not well known. Furthermore, sample-dependent nonuniform dynamics might become important as the uniform mode is excited into large angle motion just before switching, slightly modifying the switching time. To confirm the randomness of the preswitching fluctuations and coherence of postswitching oscillations 
(a)

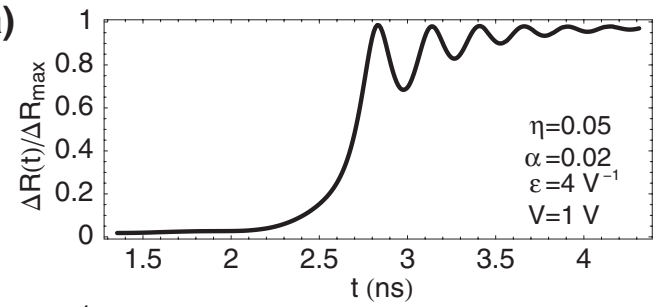

(b)

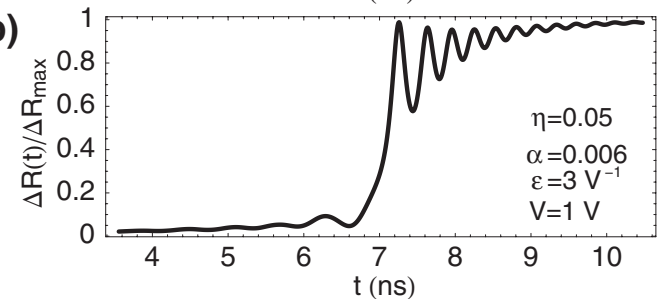

FIG. 3. (a), (b) Average of resistance traces after aligning their switching times with $\overline{t_{S}}$. In (a) $\overline{t_{S}}=2.7 \mathrm{~ns}$, while in (b) $\overline{t_{S}}=7.1 \mathrm{~ns}$.

we performed the following averaging. From the full sample of 1000 trajectories we picked the ones with switching time $t_{S}$ in the interval $\overline{t_{S}} \pm \delta t_{S}$ with $\delta t_{S}$ being the standard deviation. We then shifted all resistance traces to the same switching time $\overline{t_{S}}$ and averaged them. The result [Fig. 2(c)] shows that the postswitching ringing is indeed preserved but the random fluctuations that precede magnetization reversal are averaged out. It also shows that the transition from a slow to a sharp resistance increase, which occurs close to $0.2 \Delta R$, is preserved. The inset to Fig. 2(c) allows comparison of this result with the typical switching process induced by Slonczewski's spin torque alone $(\epsilon=0)$. In the latter case one observes a clear build up of the precession amplitude preceding magnetization reversal, while the postswitching oscillations are reduced.

We tested the sensitivity of our observations to changes in the parameters $\eta, \alpha$, and $\epsilon$. Figure 3 shows averaged resistance traces for two sets of values of the parameters $\eta, \alpha$, and $\epsilon$, illustrating that a wide range of these parameters leads to the same general behavior. As long as the efficiency is small enough so that Slonczewski's spin torque is comparable to the random thermal torques (this condition relates $\eta$ and $\alpha$ ), the behavior of the resistance is well described by Fig. 3(a). Even in the case where $\mathrm{P}$ becomes an energy maximum (for large values of $\epsilon$ and $\eta$ ) we observe similar behavior, although the average switching times are well below 1 $\mathrm{ns}$, and the probability of having an initial orientation of $\mathbf{M}$ with a switching time of more than $1 \mathrm{~ns}$ is negligibly small. However, as long as $\epsilon$ is chosen so that the P-AP energy barrier is a few times larger than the thermal energy, it is possible to obtain a wide range of switching times, even exceeding the value of $5.3 \mathrm{~ns}$ measured in Ref. 14 [Fig. 3(b)]. We observe that as the average switching times increase due to a larger P-AP barrier, the amplitude of the random preswitching fluctuations increases since larger fluctuations in $\theta$ (and therefore in resistance) are required to overcome the barrier. As shown in Fig. 3(b) some preswitching oscillations still remain after averaging, but their amplitude is much smaller than the amplitude of the postswitching oscillations. Therefore, there exists a large range of reasonable values for the parameters $\eta, \alpha$, and $\epsilon$, which satisfy the

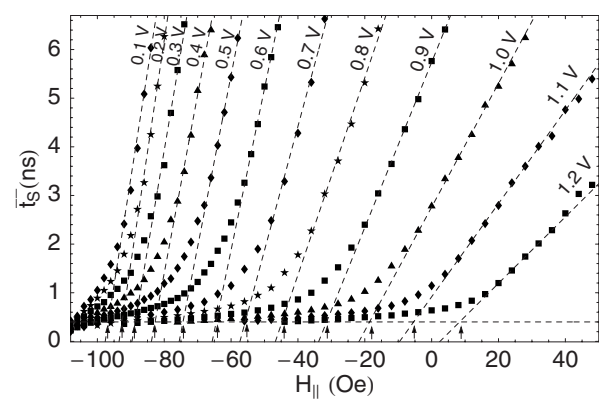

FIG. 4. Average switching time as a function of applied field for voltages between 0.1 and $1.2 \mathrm{~V}$ in $0.1 \mathrm{~V}$ increments. The horizontal dashed line shows the negative field saturation value, while the tilted dashed lines are guides to the eyes.

conditions described above and reproduce the main experimental observations.

In the present device geometry an application of an external magnetic field $H_{\|}$along the magnetic easy axis effectively modifies the fieldlike torque constant $b_{J} \rightarrow b_{J}-H_{\|}$leaving the $a_{J}$ unchanged. Experiments with separate control of the MTJ voltage $V$ and applied field $H_{\|}$can provide valuable information on the spin-transfer torque parameters. The behavior of the average switching time predicted from simulations is shown in Fig. 4. We used the same parameters as in Fig. 2 but varied the voltage between 0.1 and $1.2 \mathrm{~V}$ in steps of $0.1 \mathrm{~V}$ and applied magnetic fields between -108 and $50 \mathrm{Oe}$ along the easy axis. Positive fields favor the $\mathrm{P}$ state. To understand these simulations we note that both applied field and voltage affect the energy barrier, with $b_{J}$ decreasing and $H_{\|}$increasing it. Since $a_{J}=\left(\hbar \eta / 2 e v M_{s}\right) V / R$ where $v$ is the free layer volume, and $R$ is the effective device resistance, we get $b_{J}=\beta V|V|$ with $\beta=\hbar \eta \epsilon / 2 e v M_{S} R$. The barrier is completely eliminated at $H_{K}+H_{D}+H_{\|}-b_{J}=0$ which gives a crossover field,

$$
H_{\| *}=\beta V|V|-H_{K}-H_{D},
$$

(marked by arrows in Fig. 4) separating the ultrafast and normal switching regimes. Although the crossover is broad due to finite temperature, one would be able to extract $H_{\| *}$ from experimental data by extrapolating the dependencies $\overline{t_{S}}\left(H_{\|}\right)$above and below the crossover. Then plotting $H_{\| *}$ as a function of $V|V|$ one can find the coefficient $\beta$ and extract the value of $\epsilon$, i.e., the relative strength of the fieldlike spin torque term. To demonstrate the feasibility of such data analysis we fitted the simulated data and obtained $\epsilon$ $\approx 1.55 V^{-1}$ for a data set generated with $\epsilon=1.7 V^{-1}$, in reasonable agreement. The accuracy of $\beta$ obtained in this way depends on the accuracy of the voltage dependence of $H_{K}$ and $H_{D}$ used in Eq. (3). However, previous measurements have shown that the voltage dependence of the fieldlike torque dominates over the voltage dependence of $H_{K}{ }^{6}$ and thus as a first approximation $\beta$ can be obtained by assuming that $H_{K}$ and $H_{D}$ are voltage independent. We point out that as the external magnetic fields approach $-H_{K}-H_{D}$ (in our simulations $-108 \mathrm{Oe}$ ) the device might switch thermally even without applying any voltage. This might limit the field range over which the switching time can be measured. How- 
ever, if the approach of Devolder et $a l .{ }^{14}$ is followed, the initial state of the device is known after each transmitted pulse is observed, and thus events where the magnetization had already switched before the pulse can be discarded. In addition, if an additional long "stabilizing" pulse with opposite polarity is applied before the switching pulse, thermally induced switching around $-H_{K}-H_{D}$ can be suppressed. In this way the switching time can be measured even as the energy barrier becomes negligible. Finally, we also performed similar simulations in the absence of the fieldlike torque and observed a completely different behavior: there is no crossover between ultrafast and normal switching regimes but instead all of the curves (for different voltages) meet at $H_{\|}=-H_{K}-H_{D}$. Therefore the existence of the fieldlike torque can be observed even without fitting the switching time data to any model. Our proposed method should allow both verification of the existence of the fieldlike torque and determination of its voltage dependence, in particular the crossover between the low and high voltage regimes.
In conclusion, we show that by considering an additional fieldlike spin torque of magnitude similar to Slonczewski's spin torque, a macrospin description of MTJ switching reproduces the incubation delay, the lack of coherent magnetization precession before reversal, and the postswitching oscillations observed in Ref. 14. Our analysis suggests an additional experimental procedure to measure the voltage dependence of the fieldlike spin torque term: an external magnetic field can be applied along the easy axis to cancel or reinforce the fieldlike spin torque, while the average switching time in response to voltage pulses of different amplitudes is measured. In contrast to previous studies based on careful analysis of the antisymmetric component of ferromagnetic resonance spectra, or fits of the critical switching voltage to the spin torque model, our proposed measurements can provide a more direct access to the fieldlike spin torque term, clarify its origin, and obtain its voltage dependence in a wider range.
${ }^{1}$ J. C. Slonczewski, J. Magn. Magn. Mater. 159, L1 (1996).

${ }^{2}$ L. Berger, Phys. Rev. B 54, 9353 (1996).

${ }^{3}$ S. Petit, C. Baraduc, C. Thirion, U. Ebels, Y. Liu, M. Li, P. Wang, and B. Dieny, Phys. Rev. Lett. 98, 077203 (2007).

${ }^{4}$ H. Kubota et al., Nat. Phys. 4, 37 (2008).

${ }^{5}$ J. C. Sankey, Y.-T. Cui, J. Z. Sun, J. C. Slonczewski, R. A. Buhrman, and D. C. Ralph, Nat. Phys. 4, 67 (2008).

${ }^{6}$ Z. Li, S. Zhang, Z. Diao, Y. Ding, X. Tang, D. M. Apalkov, Z. Yang, K. Kawabata, and Y. Huai, Phys. Rev. Lett. 100, 246602 (2008).

${ }^{7}$ C. Heide, Phys. Rev. Lett. 87, 197201 (2001).

${ }^{8}$ Y. Tserkovnyak, A. Brataas, and G. E. W. Bauer, Phys. Rev. Lett. 88, 117601 (2002).

${ }^{9}$ Y. Tserkovnyak, A. Brataas, G. E. W. Bauer, and B. I. Halperin, Rev. Mod. Phys. 77, 1375 (2005).

${ }^{10}$ K. Xia, P. J. Kelly, G. E. W. Bauer, A. Brataas, and I. Turek, Phys. Rev. B 65, 220401(R) (2002).

${ }^{11}$ M. A. Zimmler, B. Özyilmaz, W. Chen, A. D. Kent, J. Z. Sun, M.
J. Rooks, and R. H. Koch, Phys. Rev. B 70, 184438 (2004).

${ }^{12}$ I. Theodonis, N. Kioussis, A. Kalitsov, M. Chshiev, and W. H. Butler, Phys. Rev. Lett. 97, 237205 (2006).

${ }^{13}$ J. C. Slonczewski and J. Z. Sun, J. Magn. Magn. Mater. 310, 169 (2007).

${ }^{14}$ T. Devolder, J. Hayakawa, K. Ito, H. Takahashi, S. Ikeda, P. Crozat, N. Zerounian, J.-V. Kim, C. Chappert, and H. Ohno, Phys. Rev. Lett. 100, 057206 (2008).

${ }^{15}$ J. Z. Sun, Phys. Rev. B 62, 570 (2000).

${ }^{16}$ I. N. Krivorotov, N. C. Emley, J. C. Sankey, S. I. Kiselev, D. C. Ralph, and R. A. Buhrman, Science 307, 228 (2005).

${ }^{17}$ W. F. Brown, Phys. Rev. 130, 1677 (1963).

${ }^{18}$ J. L. García-Palacios and F. J. Lázaro, Phys. Rev. B 58, 14937 (1998).

${ }^{19}$ J. C. Slonczewski, Phys. Rev. B 71, 024411 (2005).

${ }^{20}$ Z. Diao, D. Apalkov, M. Pakala, Y. Ding, A. Panchula, and Y. Huai, Appl. Phys. Lett. 87, 232502 (2005). 